\title{
Automatic Detection and Correction of Blink Artifacts in Single Channel EEG Signals
}

\author{
G Bhaskar N Rao ${ }^{1}$ \\ Department of Commerce MAHE \\ Manipal University \\ Kasturba Nagar \\ Manipal - 576104
}

\author{
Dr. Anumala Vijay Sankar ${ }^{2}$ \\ ECE Dep. Associate Professor \\ DE \& CS Dept of ECE VRSEC \\ Chalasani Nagar, Kanuru \\ Vijayawada, AP-520007
}

\author{
Dr. Peri Pinak Pani ${ }^{3}$ \\ GITAM HBS \\ Rudraram, Patancheru \\ Sanga Reddy \\ Dist. TS - 502329
}

\author{
Aneesh Sidhireddy ${ }^{4}$ \\ ECE - IV Year \\ Roll No: 16BEC0690 \\ VIT University, Vellore, 632014 \\ Tamil Nadu, India
}

\begin{abstract}
Ocular Artifacts (OAs) are inevitable during EEG acquisition and make the signal analysis critical. Detection and correction of these artifacts is a major problem now a day's. In this paper an energy detection method is used to detect the artifacts and performed wavelet thresholding within the researched zones to protect neural data at non blink regions. Various sets of Wavelet Transform (WT) techniques and threshold functions are collated and identification of the optimum combination for OA's separation is indicated in many research areas including Technology $\&$ Management. The output of these methods at blink regions is compared interns of various standard metrics using established techniques of Supply Chains. Results of this study demonstrate that the SWT+HT has better in rejecting the artifacts than other methods in this paradigm.
\end{abstract}

Keywords-Electroencephalogram (EEG); ocular artifacts; wavelet transform; hybrid threshold

\section{INTRODUCTION}

EEG is a non-invasive technique used to diagnose brain related diseases and disorders. These signals are frequently corrupted by various types of artifacts during acquisition that come from several sources such as blinking of eyes, cornea movements, vibrations of muscle, heart signals can reduce the clinical utility. Among these ocular activities create significant artifacts due to its larger amplitude and makes the analysis critical. Numerous methods are in use to detect the artifacts, but WT techniques are popular due to its easier implementation [1-4]. Krishnaveni et.al [1] has proposed an artifact detection method based on the relative amplitudes of Artifact Rising Edges (ARE) and Artifact Falling Edges (AFE) at Nth decomposition level. Later it is simplified by the process of coefficient of variation. Usually each spike contains three coefficients; from the number of coefficients at each decomposition level recognize the coefficients pertinent to spikes $[2,3]$. However detection of artifacts depends on the selection of a parent wavelet function and associated decomposition quotient. WT is a proven methodology and has shown promise of its utility in ocular artifact paradigm and for single channel EEG signals [3-5]. Majmudar et.al [5] compared the discrete and stationary wavelet transforms using various methodologies on SWT and recommended that DWT was superior in artifact correction .Of late Jianbo Gao et.al proposed a Wavelet based hybrid threshold function for denoising nonlinear time signals [6]. This paper proposed an energy detector method for identification of the artifacts and performs adaptive thresholding to the blink regions for effective removal of ocular artifacts. An interpretation of experimental data using queuing theory is adopted for latency and crystallization of decisions [14].

\section{DATA ACQUISITION}

Raw EEG segments for this work are taken from physionent (www.physionet.org/ physionet/physiobank ATM/eegmmidb) [7]. EEG signals at frontal channels such as F7, F8, Fp1 and Fp2 are taken for analysis, because these electrodes are placed close to the eyes and EEG signals are most likely to be affected by the ocular artifacts. Analysis is done by taking EEG segments of 10 seconds duration each, since EEG epochs smaller than 12 seconds may be considered stationary. The simulations are carried out in the MATLAB environment within the time-frequency domain.

\section{Methodology}

1) The raw EEG signal is segregated into blink and nonblink regions by energy detector method and perform wavelet denoising to the identified zones.

2) Each blink region is decomposed by WT methods into approximation and detail coefficients up to 8 levels.

3) Levels range from 8 to 4 and function as an inverse WT to reconstruct the refined EEG signal. Fig 1 illustrates the process of artifact removal of raw EEG signals. 


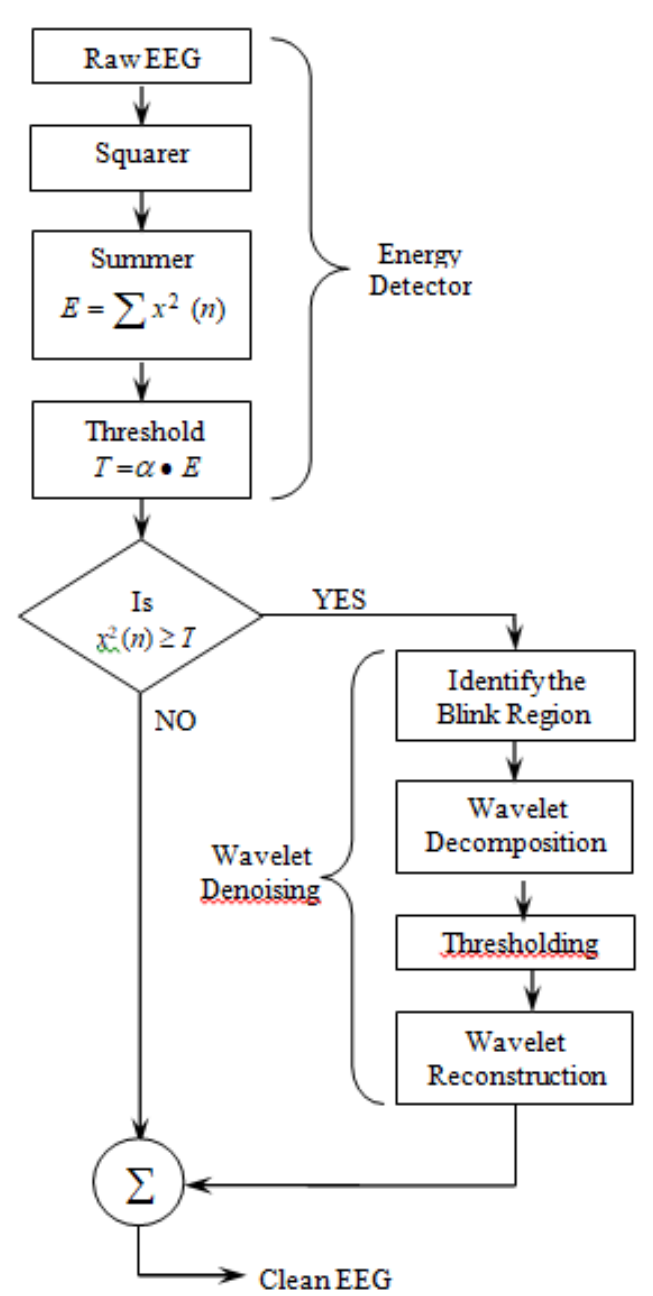

Fig. 1. Flow Chart for Detection and Correction of Blink Artifacts.

\section{ENERGY DETECTOR METHOD FOR IDENTIFICATION OF BLINK REGIONS}

Correcting the artifacts at blink regions and preserving the neural information at non-blink regions is of very much importance for clinical diagnosis. The EEG signal, tabled evenly between scalp, had amplitude of about $11 \mu \mathrm{V}$ to 101 $\mu \mathrm{V}$, whereas artifacts due to ocular activity are 10 to 100 times as that of the EEG signal. The momentous difference in magnitude between the artifacts facilitates to separate the blink and non-blink regions by an energy detector method. Energy detector is a basic signal detection method $[8,9]$. Blink and non blink regions are segregated by comparing the relative amplitudes of squarer with respective to the threshold level. Threshold level $\mathrm{T}$ is estimated from signal statistics as whereas factor $\alpha$ is ranging from 0.001 to 0.01 , which decides the accuracy of artifact detection. The detection of multiple artifacts for Fp2 EEG signal is presented below in Fig 2(a) and Fig 2(b).

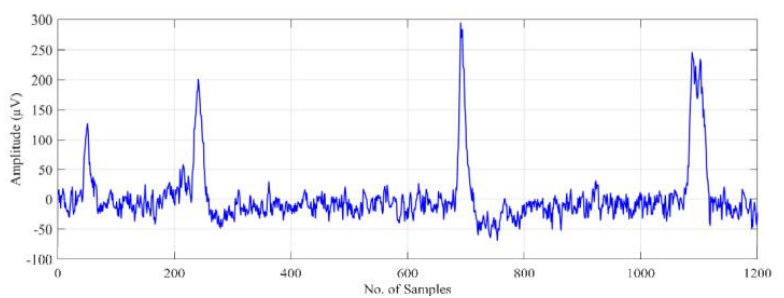

(a)

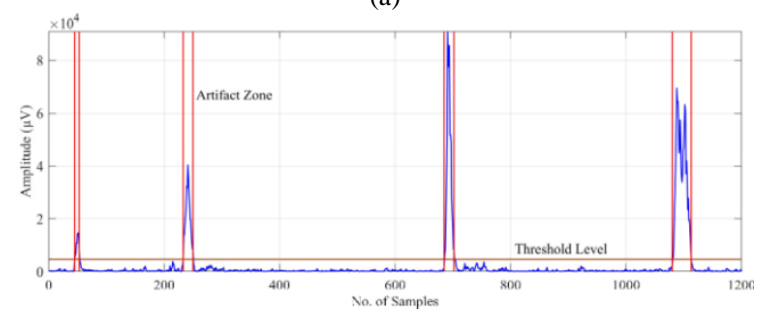

(b)

Fig. 2. (a) Fp2 EEG Signal, (b) Multiple Artifacts for Fp2 EEG Signal.

\section{Threshold FunCtions AND PERFormance METRICS}

\section{A. Thresholding Functions}

In the proposed method, the following thresholds were used for calculating the threshold function and the most optimum one is found.

a) Universal Threshold (UT): UT is a global threshold function, tabulation of values at the Threshold as per Eq. (1).

$\lambda_{l}=\sigma_{l} \sqrt{2 \log N}$

where $\mathrm{N}$ = signal measurement, and is the maximum value at $\mathrm{i}^{\text {th }}$ decomposition level. $\sigma_{\mathrm{i}}$ is the average deviation for $\mathrm{Wi}$, which is calculated by the following Eq. (2).

$\mathrm{O}_{l}=\frac{\text { Median }\left|W_{i}\right|}{0.6745}$

where $\mathrm{W}_{\mathrm{i}}=$ comprehensive wavelet coefficients at $\mathrm{i}^{\text {th }}$ level. The numerator is rescaled for a suitable estimator for Gaussian white noise by 0.6834 in the divisor.

b) Statistical Threshold (ST): ST was proposed by Krishnaveni et.al, which is based on the statistics of the signal [5].

The effective statistical threshold is given by

$\lambda=1.5 * \operatorname{std}\left(\mathrm{W}_{\mathrm{i}}\right)$

in which factor 1.5 is an estimator for standard white Gaussian noise

c) Hybrid Threshold (HT): HT is a combination of UT and ST functions [6], Threshold function at each level is defined by

$\lambda_{l}=\operatorname{std}\left(\mathrm{W}_{\mathrm{i}}\right) * \sqrt{2 \log N}$ 


\section{B. Performance Metrics}

Working of the threshold functions is validated using power spectral density (PSD), Magnitude Square Coherence (MSC) plots and twin statistical parameters: Artifact Rejection Ratio (ARR) and Correlation coefficient (CC). ARR is the power ratio of the removed artifacts to the clean EEG signals expressed [10].

$A R R=\frac{\sum_{n=1}^{N}(\mathrm{x}[\mathrm{n}]-y[n])^{2}}{\left.\sum_{n=1}^{N} y[n]\right)^{2}}$

Where $x[n]$ and $y[n]$ represents the contaminated and clean EEG signals. $\mathrm{CC}$ is a statistical quantity that shows the degree of similarity or relatedness between two signals expressed as

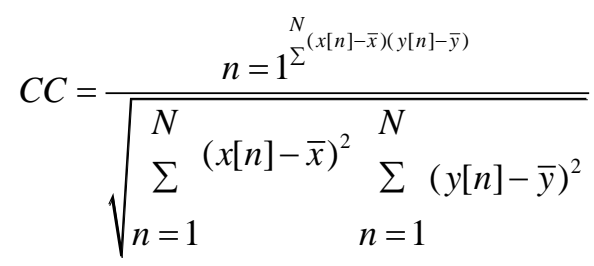

Based on the power distribution from 0 to $16 \mathrm{~Hz}$, SWT+HT has best in rejecting the artifacts, whereas DWT $+\mathrm{HT}$ is second

Where $x$ and $y$ represents the mean of raw and clean EEG signals. The Power Spectral Density (PSD) function shows the energy of the signal as a function of frequency. It uses Welch's method (Pwelch). MSC provides the estimate of the frequency coherence between the two signals, which is implemented using 'MScohere' MATLAB function.

\section{RESULTS AND DisCUSSIONS}

Denoising of EEG signal is carried by combination wavelet transform methods and threshold functions. Fig. 3 (a) to 3(d) shows the time domain plots of the raw and clean EEG signals using various methods. By visual inspection it is clear that SWT method is superior in correcting the artifacts than DWT method. Threshold function HT is better than other functions in both the methods, whereas ST is the second best. Fig. 4 illustrates the power spectra of DWT and SWT methods using different threshold functions. Threshold functions HT and ST have provided the minimum power at lower frequencies respectively in both the methods [11] and [12]. The MSC plot for FP1 EEG signal is shown in Fig. 5 and Fig. 6, it is observed that the frequency coherence is less at lower frequencies and nearly ' 1 ' for all higher frequencies in both the methods [11].

The blink and non-blink regions are segregated using energy detector method and perform thresholding to the blink regions alone to preserve the neural information at non OAs zone. Table 1 delineates the performance metrics of various artifact removal methods over blink regions

Improvement in ARR specifies the extent to which artifacts are removed from the original EEG signal and the improvement in $\mathrm{CC}$ indicates the similarity are relatedness between the raw and clean EEG signals during blink regions.
An effective artifact removal method should maintain high ARR and poor CC between the raw and clean EEG signals over the blink regions. From Table 1, it is observed that SWT is exceptionally good, whereas DWT is more applicable next to SWT.

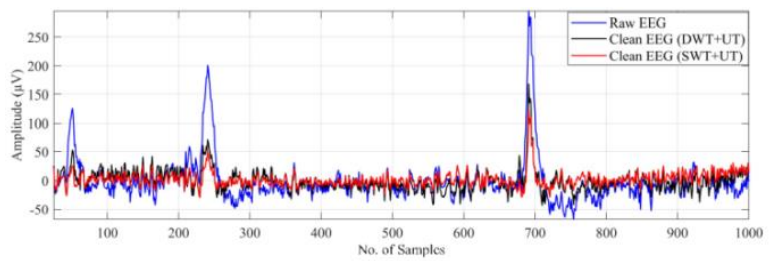

(a)

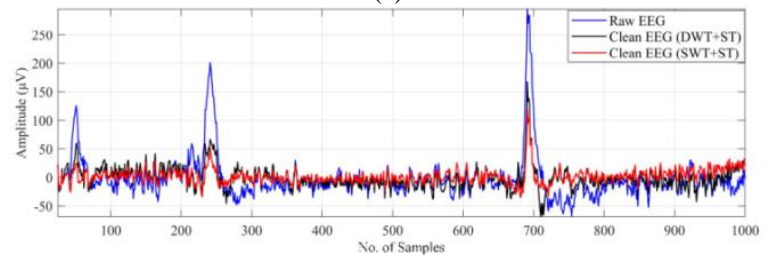

(b)

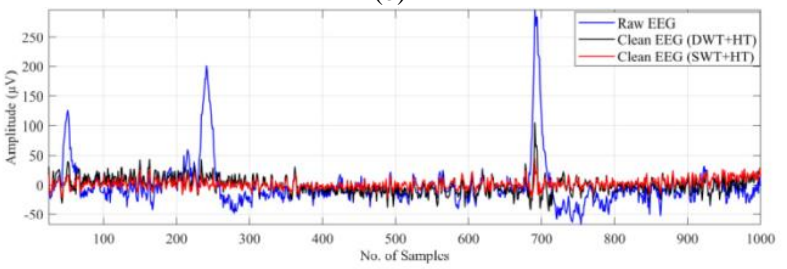

(c)

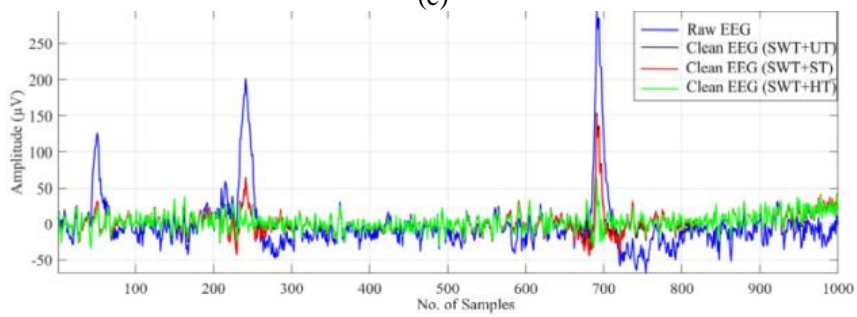

(d)

Fig. 3. (a) EEG Signal Extraction using Time Frequency Distributions (TFD), (b) EEG Signal Extraction using Fast Fourier Transform (FFT), (c) EEG Signal Extraction using Eigenvector Methods (EM), (d) EEG Signal Extraction using Stationary Wavelet Transform (SWT).

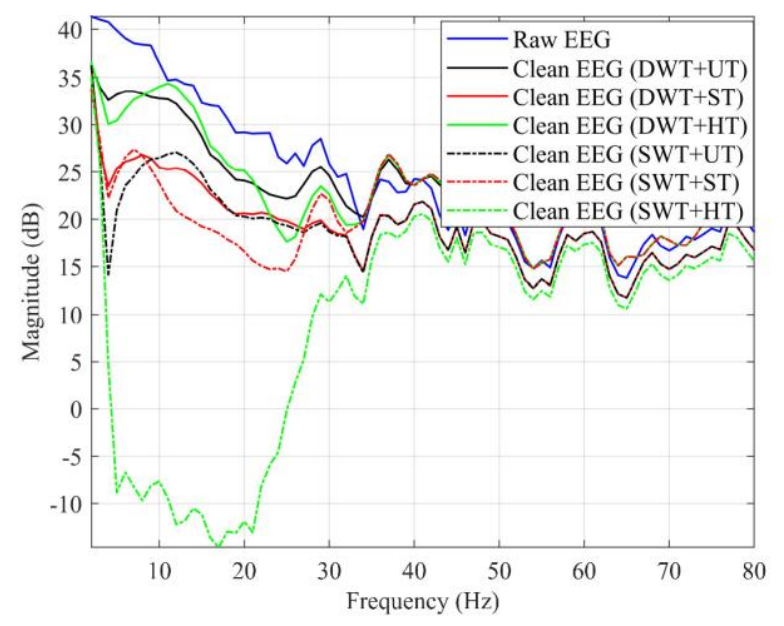

Fig. 4. Signal Analysis using Wavelet Theory DWT, UT, ST, SWT \& HT. 
TABLE. I. ARR AND CC BETWEEN RAW AND CLEAN EEG SignALS OVER BLINK REGIONS By WT METHOdS

\begin{tabular}{|c|c|c|c|c|c|c|c|c|c|c|}
\hline \multirow{2}{*}{ Channel } & \multirow{2}{*}{ Method } & \multirow{2}{*}{$\begin{array}{l}\text { Threshold } \\
\text { Function }\end{array}$} & \multicolumn{2}{|c|}{ Blink1 } & \multicolumn{2}{|c|}{ Blink2 } & \multicolumn{2}{|c|}{ Blink3 } & \multicolumn{2}{|c|}{ Blink4 } \\
\hline & & & ARR & $\mathrm{CC}$ & ARR & $\mathrm{CC}$ & ARR & $\mathrm{CC}$ & ARR & $\mathrm{CC}$ \\
\hline \multirow{6}{*}{ F7 } & \multirow{3}{*}{ DWT } & UT & 3.22 & 0.422 & 3.62 & 0.362 & 4.17 & 0.245 & 4.94 & 0.236 \\
\hline & & ST & 3.84 & 0.384 & 4.26 & 0.286 & 4.64 & 0.228 & 5.16 & 0.198 \\
\hline & & HT & 5.98 & 0.312 & 6.14 & 0.243 & 6.4 & 0.212 & 6.84 & 0.175 \\
\hline & \multirow{3}{*}{ SWT } & UT & 3.92 & 0.254 & 4.54 & 0.238 & 4.84 & 0.193 & 5.18 & 0.198 \\
\hline & & ST & 4.48 & 0.214 & 5.22 & 0.205 & 5.24 & 0.171 & 5.56 & 0.156 \\
\hline & & HT & 6.26 & 0.152 & 6.98 & 0.118 & 7.22 & 0.142 & 7.6 & 0.122 \\
\hline \multirow{6}{*}{ F8 } & \multirow{3}{*}{ DWT } & UT & 2.82 & 0.558 & 3.3 & 0.453 & 3.37 & 0.362 & 4.14 & 0.272 \\
\hline & & $\mathrm{ST}$ & 3.87 & 0.514 & 3.64 & 0.358 & 3.94 & 0.25 & 4.82 & 0.192 \\
\hline & & HT & 5.48 & 0.36 & 6.24 & 0.286 & 5.72 & 0.196 & 6.24 & 0.128 \\
\hline & \multirow{3}{*}{ SWT } & UT & 3.08 & 0.428 & 3.76 & 0.34 & 3.76 & 0.298 & 4.86 & 0.224 \\
\hline & & ST & 4.42 & 0.415 & 4.86 & 0.307 & 4.92 & 0.227 & 5.84 & 0.182 \\
\hline & & HT & 6.4 & 0.294 & 7.18 & 0.196 & 7.45 & 0.143 & 8.11 & 0.116 \\
\hline \multirow{6}{*}{ Fp1 } & \multirow{3}{*}{ DWT } & UT & 2.54 & 0.485 & 3.18 & 0.407 & 3.82 & 0.305 & 4.48 & 0.257 \\
\hline & & ST & 3.82 & 0.398 & 4.38 & 0.358 & 4.42 & 0.283 & 5.2 & 0.212 \\
\hline & & HT & 6.66 & 0.296 & 6.8 & 0.254 & 7.15 & 0.182 & 7.42 & 0.136 \\
\hline & \multirow{3}{*}{ SWT } & UT & 3.66 & 0.412 & 4.96 & 0.345 & 5.18 & 0.275 & 5.52 & 0.214 \\
\hline & & ST & 4.62 & 0.312 & 5.44 & 0.296 & 5.84 & 0.242 & 6.88 & 0.197 \\
\hline & & HT & 5.87 & 0.222 & 6.98 & 0.214 & 7.44 & 0.147 & 7.98 & 0.132 \\
\hline \multirow{6}{*}{$\mathrm{Fp} 2$} & \multirow{3}{*}{ DWT } & UT & 2.42 & 0.428 & 2.78 & 0.363 & 3.25 & 0.242 & 4.92 & 0.218 \\
\hline & & ST & 3.44 & 0.384 & 4.1 & 0.228 & 4.42 & 0.165 & 5.4 & 0.156 \\
\hline & & HT & 6.37 & 0.21 & 7.44 & 0.166 & 7.82 & 0.143 & 8.22 & 0.134 \\
\hline & \multirow{3}{*}{ SWT } & UT & 4.24 & 0.325 & 4.82 & 0.23 & 5.12 & 0.218 & 5.28 & 0.207 \\
\hline & & $\mathrm{ST}$ & 4.98 & 0.294 & 5.52 & 0.217 & 5.84 & 0.196 & 6.14 & 0.164 \\
\hline & & HT & 6.54 & 0.163 & 6.98 & 0.146 & 7.84 & 0.125 & 8.45 & 0.118 \\
\hline
\end{tabular}

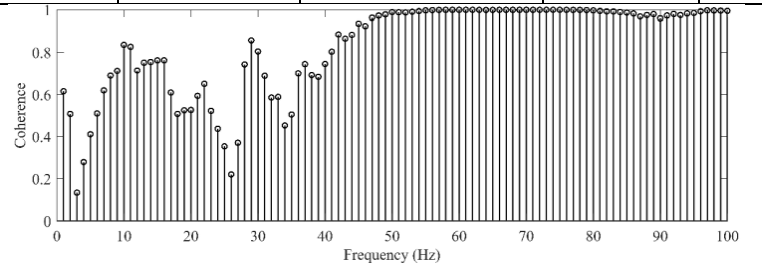

Fig. 5. Power Spectra of Raw and Clean EEG Signals by DWT and SWT Methods using Various Threshold Functions.

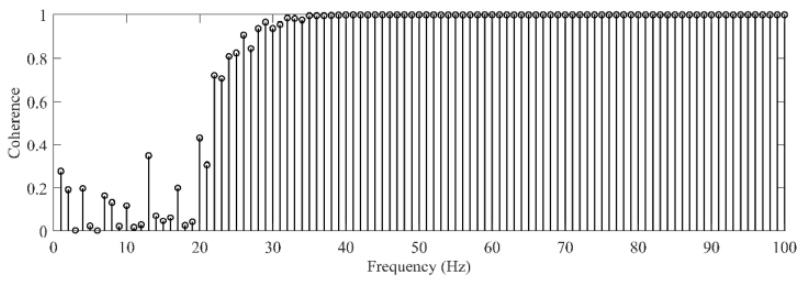

Fig. 6. Magnitude Square Coherence for Fp1 EEG Signal by a) DWT+HT b) SWT+HT.

However, threshold function HT is the best in both the methods and ST is the second best.
The average execution time required to blink region by different methods is given in presented below in Table 2 [13]. The results might be due to its larger redundancy at each decomposition level.

TABLE. II. ARR AND CC BETWEEN RAW AND CLEAN EEG SigNALS OVER BLINK REGIONS BY WT METHODS

\begin{tabular}{|l|l|l|}
\hline Method & DWT & SWT \\
\hline Average Execution Time (Sec) & 0.025 & 0.275 \\
\hline
\end{tabular}

\section{VII.CONCLUSIONS}

In this manuscript, a Hybrid method is proposed for detection and correction of blink artifacts in single channel EEG signals. Due to finite energy difference between EEG and blink artifacts, Energy Detection method should be an optimum choice for detection of blink artifacts at various levels of EEG signal. The efficacy of the WT methods using various threshold functions are compared in terms of metrics ARR and CC during blink regions. It was observed that SWT method has shown superior performance than DWT method, and threshold function HT is better than other threshold functions in both the methods. SWT $+\mathrm{HT}$ method is 
exceptionally good in rejecting the artifacts but time consuming. Hence, DWT $+\mathrm{HT}$ is a better choice for correction of OAs for real time application whereas SWT+HT is the better choice for offline applications.

\section{REFERENCES}

[1] V.Krishnaveni, S.Jayaraman, S.Aravind, V.Hariharasudhan, K.Ramadoss, "Automatic Identification and Removal of Ocular Artifacts from EEG using Wavelet Transform", MEASUREMENT SCIENCE REVIEW, Volume 6, Section 2, No. 4, 2006, pp. 45-57.

[2] P. Senthil Kumar, R. Arumuganathan, K. Sivakumar, C. Vimal, "Removal of Ocular Artifacts in the EEG through Wavelet Transform without using an EOG Reference Channel", Int. J. Open Problems Compt. Math., Vol. 1, No. 3, December 2008, pp. 188-200.

[3] G.Geetha, Dr.S.N.Geethalakshm, "De-Noising Of EEG Signals Using Bayes Shrink Based on Coiflet Transform", Proc. of Int. Conf on Advances in Recent Technologies in Communication and Computing 2011, IET, pp. 41-46.

[4] Qinglin Zhao, Bin Hu, Yujun Shi, Yang Li, Philip Moore, Minghou Sun, Hong Peng, "Automatic Identification and Removal of Ocular Artifacts in EEG-Improved Adaptive Predictor Filtering for Portable Applications", IEEE Transactions on Nano-Bioscience, Vol.13, No.2, June 2014, pp. 109-117.

[5] Charvi A. Majmudar, Ruhi Mahajan, and Bashir I Morshed, "RealTime Hybrid Ocular Artifact Detection and Removal for Single Channel EEG", 2015 IEEE International Conference on Electro/Information Technology (EIT), 21-23 May 2015, pp. 330334.

[6] Jianbo Gao, Member, IEEE, Hussain Sultan, Jing Hu, Member, IEEE, and Wen-Wen Tung, "Denoising Nonlinear Time Series by Adaptive Filtering and Wavelet Shrinkage: A Comparison", IEEE Signal Processing Letters, Vol. 17, No. 3, March 2010, pp. 237-240.

[7] EEG Motor Movement/Imagery Dataset (eegmmidb).[Online]. Available: https://physionet.org/cgi-bin/atm/ATM, accessed 2018

[8] Harry Urkowitz, "Energy Detection of Unknown Deterministic Signals", PROCEEDINGS OF THE IEEE, APRIL 1967, pp. 523531.
[9] Fadel F. Digham, Mohamed-Slim Alouini, MarvinK. Simon, "On the Energy Detection of Unknown Signals Over Fading Channels", IEEE Transactions on Communications, Vol. 55, No. 1, January 2007, pp. 21- 24.

[10] Hossein Shahabi, Sahar Moghimi, Hossein Zamiri-Jafarin, "EEG Eye Blink Artifact Removal by EOG Modeling and Kalman Filter", BMEI 2012, pp. 496-500.

[11] D. Hamidian, J. Salajegheh, E. Salajegheh, "Damage Detection of Irregular Plates and Regular Dams by WaveletTransform Combined Adoptive Neuro Fuzzy Inference System", Civil Engineering Journal, 2018, Vol 4, No.2, pp. 305-319, DOI: http://dx.doi.org/10.28991/cej- 030993

[12] Drapaca C.S., "Mathematical Modeling of a Brain-on-a-Chip: A Study of the Neuronal Nitric Oxide Role in Cerebral Microaneurysms", Emerging Science Journal, Vol 2, No 6 (2018), DOI: $10.28991 /$ esj- 2018-01156

[13] Espejel-García D, Luis Ricardo Ortíz-Anchondo, Cornelio AlvarezHerrera, Alfonso Hernandez-López, Vanessa Verónica EspejelGarcía, Alejandro Villalobos-Aragón., "An Alternative Vehicle Counting Tool Using the Kalman Filter within MATLAB", Civil Engineering Journal, Vol 3, No 11 (2017), DOI: 10.28991/cej030935

[14] Garimella, Bhaskar \& Shenoy, Sandeep \& Pai, Rashmi \& Shetty, Rishali. (2018). Structured Education for Sustainable Employment: Technology Enabled Queueing Theory Applications. Foundations of Management. 10. 75-86. 10.2478/fman-2018-0007. 